\title{
The Magnetic Properties of Closely Spaced Three-Dimensional Nanomagnet Array
}

\author{
Ying Li, ${ }^{1}$ Tianxing Wang, ${ }^{2}$ Heyan Liu, ${ }^{1}$ Xuefang Dai, ${ }^{1}$ Xiao Yu, ${ }^{1}$ and Guodong Liu ${ }^{1}$ \\ ${ }^{1}$ School of Materials Science and Engineering, Hebei University of Technology, Tianjin 300130, China \\ ${ }^{2}$ College of Physics and Information Engineering, Henan Normal University, Xinxiang 453007, China \\ Correspondence should be addressed to Ying Li; liyingphy@126.com
}

Received 30 June 2015; Accepted 30 August 2015

Academic Editor: Fei Meng

Copyright (c) 2015 Ying Li et al. This is an open access article distributed under the Creative Commons Attribution License, which permits unrestricted use, distribution, and reproduction in any medium, provided the original work is properly cited.

\begin{abstract}
With Monte Carlo method, we investigate the magnetic ground state, magnetic specific heat, and magnetic hysteresis loop for three types of closely spaced nanomagnet arrays in three-dimensional (3D) space. It is found that the magnetic ground state of three nanomagnet arrays exhibits the vortex order, caused by the long-range dipolar interactions. Three types of nanomagnet arrays have almost the same magnetic transition temperature even if their array formation in 3D triangular lattice is different. Some slight jump occurs in the hysteresis loop of the face-centered cubic nanomagnet array, while for the simple hexagonal nanomagnet and close-packed hexagonal nanomagnet arrays no jump is found.
\end{abstract}

\section{Introduction}

The nanomagnet assembly with various arrays received much attention because of its importance for magnetoelectronic devices such as read head sensor, magnetic random access memory, and magnetic resonance imaging [1] and also for emerging technologies such as spin logic gates $[2,3]$ and magnonic crystals [4]. Currently, the key issue from nanomagnet application is to understand and control the magnetic properties of individual and interacting nanomagnet elements. Individual nanomagnet has a limitation for the permanent information storage due to its random thermal fluctuation at high temperature. By comparison, the nanomagnet assembly has an enhanced thermal stability owing to strong magnetostatic interaction between nanomagnets and hence exhibits a promising application in nanomagnetic logic architecture [5]. The nanomagnetic logic circuits process information by manipulating the magnetization states of single-domain nanomagnets coupled with their nearest neighbors through magnetic dipole interactions. It is therefore very important to investigate the magnetic properties of the dipolar-coupled nanomagnets.

Experimentally, the nanomagnet array can be realized by conventional electron-beam lithography $[6,7]$. For one-dimensional (1D) or two-dimensional (2D) array of nanomagnets, the magnetic properties have been extensively investigated experimentally or theoretically in the past decades [8-14]. However, the three-dimensional (3D) nanomagnet array received a little attention until recently due to the difficulty of fabrication in experiment [15]. In this case, a numerical simulation using a Monte Carlo algorithm is an appropriate way of predicting the magnetic behavior. For assembly of nanoparticles embedded in nonmagnetic matrix systems, the exchange interactions between particles are approximately negligible and the dipolar interactions can be crucial in determining the magnetic states at low temperatures and the magnetization properties in the external field. Different magnetic ordering may occur depending on the array formation of nanoparticle as well as the magnetic dipolar interaction strength between nanoparticles.

In present work, we simulate the magnetic ground state, the magnetic transition, and hysteresis loop of 3D array of the nanomagnet located at the triangle lattice sites with the Monte Carlo method. In contrast to the 2D nanomagnet array $[8,11]$, the $3 \mathrm{D}$ nanomagnet array in the triangular lattice has three kinds of array formation depending on the order of staggered layers, and different arrays may lead to different frustration magnetic properties. It is found that the magnetic 


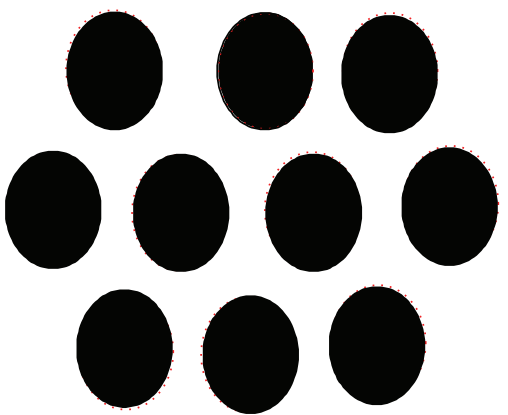

(a)

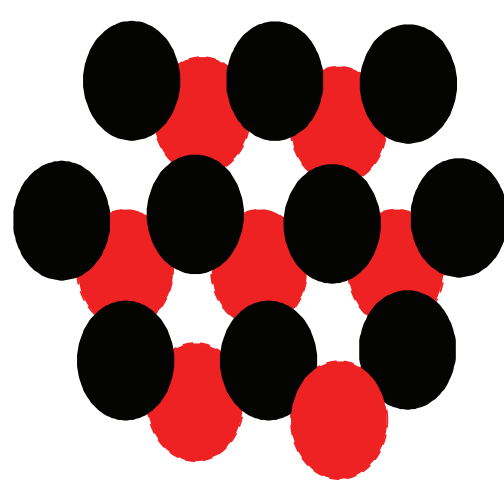

(b)

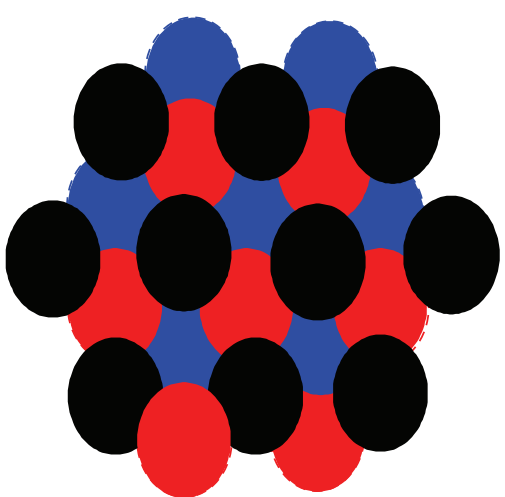

(c)

FIgURE 1: The two-dimensional projection demonstration of three types of nanomagnet assembly on the $x y$ plane: (a) simple hexagonal nanomagnet array, (b) hexagonal close-packed nanomagnet array, and (c) face-centered cubic nanomagnet array.

configurations exhibit a vortex order for three types of nanomagnet arrays but different vortex direction. The magnetic transition temperature and magnetization hysteresis loop have also some difference from three types of nanomagnet arrays.

\section{Model and Computational Details}

As described often in experimental studies [7], a magnetic nanoparticle with a given diameter and shape will have a single-domain state and can be viewed as a giant spin. The value of diameter usually depends on the composition and shape of nanoparticle [16]. Here, assume that nanoparticles with the single-domain state are arranged regularly at sites of the triangular lattice in a 3D space to form nanomagnet assembly, and they are magnetically very soft and hence have a negligible magnetic anisotropy, such as the FePd or $\mathrm{NiFe}$ permalloy [6]. Consider three types of array formations of nanomagnets. The basic units of array formation out of the plane are displayed in Figure 1 for three nanomagnet assemblies, respectively. The first type is the simple hexagonal array (SHA) where each layer is located directly on its adjacent layer (Figure 1(a)). The second type is the hexagonal close-packed array (HCPA) where one staggered stacking exists between adjacent two layers (Figure 1(b)). And the third type is the face-centered cubic array (FCCA) where there exists another staggered stacking among adjacent three layers (Figure 1(c)).

The Hamiltonian energy of the nanomagnet assembly is then given by

$$
\mathscr{H}=E_{\text {ex }}+E_{\text {dip }}+E_{H}
$$

with

$$
\begin{aligned}
E_{\mathrm{ex}} & =-J \sum_{\langle i j\rangle} \vec{s}_{i} \cdot \vec{s}_{j}, \\
E_{\mathrm{dip}} & =D \sum_{\{i, j\}}\left[\frac{\vec{s}_{i} \cdot \vec{s}_{j}}{\left|\vec{r}_{i j}\right|^{3}}-\frac{3\left(\vec{s}_{i} \cdot \vec{r}_{i j}\right)\left(\vec{s}_{j} \cdot \vec{r}_{i j}\right)}{\left|\vec{r}_{i j}\right|^{5}}\right], \\
E_{H} & =-\vec{H} \cdot \sum_{i} \vec{s}_{i},
\end{aligned}
$$

where $\vec{s}_{i}\left(\vec{s}_{j}\right)$ is a three-dimensional unit vector, representing the spin at the site $i(j)$ in the lattice. The first term on the right-hand side of (1) denotes the exchange interactions with $J$ being the exchange energy parameter and the sum running over all the nearest neighboring spin pairs $i$ and $j$. Here, the intrananomagnet exchange interactions lead to the singledomain state and hence each nanomagnet can be served as a giant spin. So the exchange energy parameter is zero in our paper. The second term represents the dipolar interactions where $r_{i j}$ is the distance between spins $s_{i}$ and $s_{j}$ measured in unit of nearest-neighbor spins. $D=\mu_{0} g \mu_{B}^{2} / 4 \pi d^{3}$ is dipolar coupling parameter with $\mu_{0}$ being the permeability of the vacuum, $g$ Landé factor, $\mu_{B}$ the Bohr magneton, and $d$ the real distance of nearest-neighbor spins, and the sum runs over all spin pairs $i$ and $j$ defining the vector $\vec{r}_{i j}$. The last term is Zeeman energy from the applied field $H$. Note that here the spin $\vec{s}_{i}$ is normalized to unity, so that the field variable $H$ as well as the dipolar coupling parameter has units of energy.

In the calculation, all three types of nanomagnet assembly contain 1728 nanomagnet elements, and the magnetic moment of each nanomagnet is about $10^{6} \mu_{B}$. The dipolar coupling parameter is taken as $3.62 \mathrm{eV}$ unless otherwise stated, corresponding to the case of closely spaced nanomagnet array. By Monte Carlo method [17, 18], we simulate the magnetic configurations at the ground state and magnetic phase transition for three different nanomagnet assemblies. The long-range dipolar interactions are considered without any energy cutoff. Our simulation uses a single-spin flip algorithm under free boundary conditions [17-19]. Start from an initial random magnetic state at very high temperature, and end with a final ground state when the temperature is decreased to $0.01 \mathrm{D}$ with temperature interval of $0.01 \mathrm{D}$. Moreover, the hysteresis loop is simulated for two different space distances, that is, two different dipolar coupling parameters. To simulate the magnetization reversal behavior, as described in our previous work [14], the magnetic field is first applied and increased to a certain field $H_{s}$ where the system reaches the saturated state. After saturating the magnetization in the positive direction, the field is subsequently reduced to zero and increased to $-H_{s}$ in steps of $0.01 H_{s}$ and then increased 


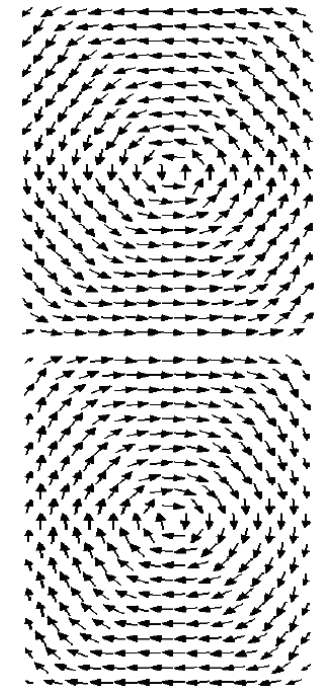

(a)

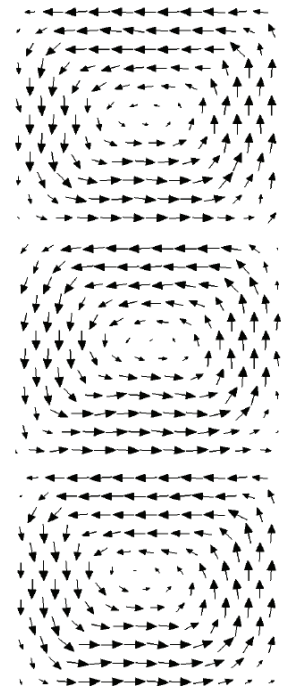

(b)

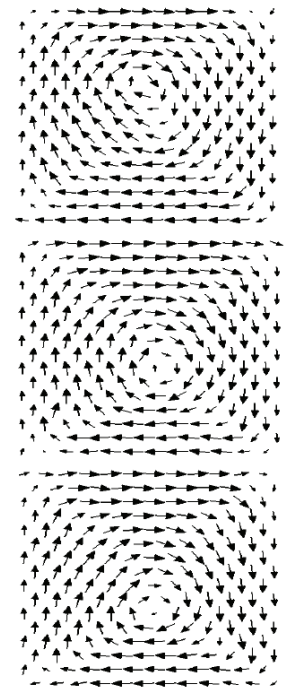

(c)

FIGURE 2: The snapshots of ground state spin configurations for the nanomagnet assembly with simple hexagonal array (a), hexagonal closepacked nanomagnet array (b), and face-centered cubic nanomagnet array (c).

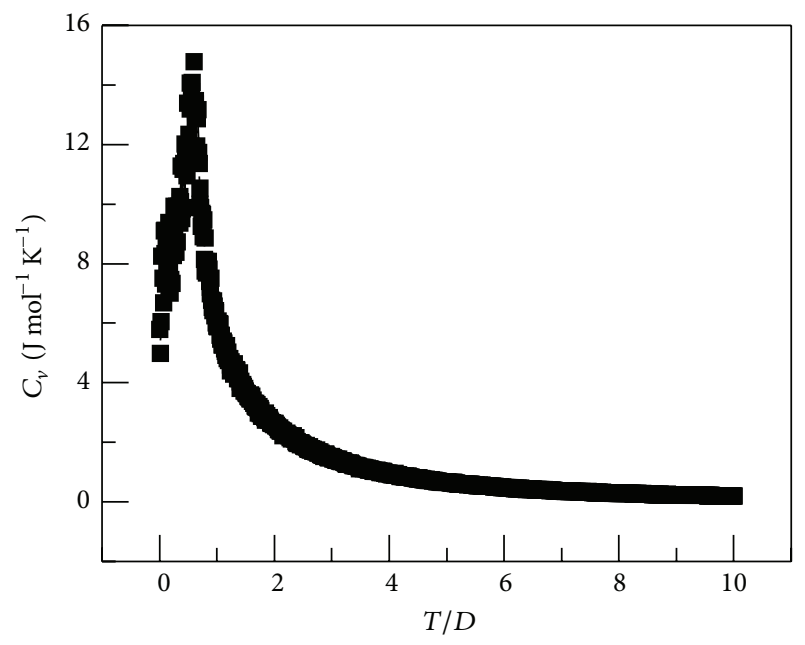

(a)

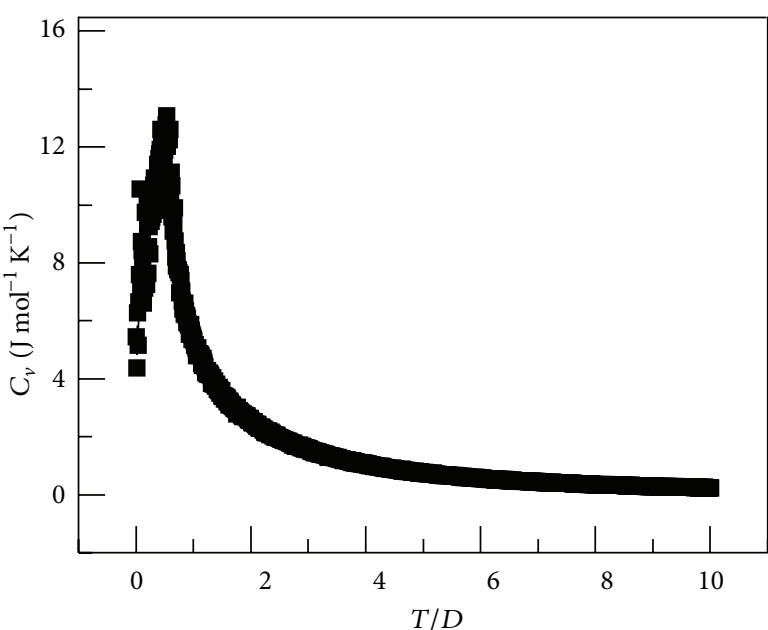

(b)

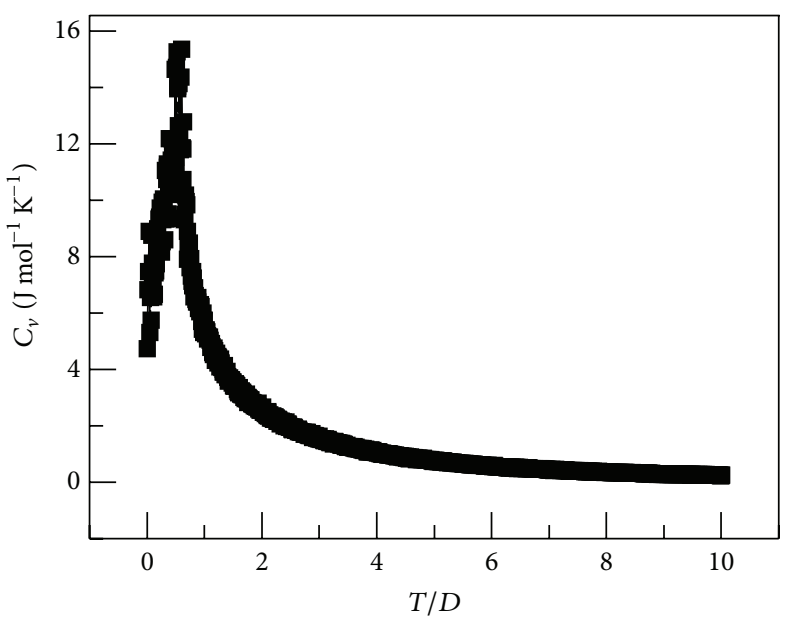

(c)

FIgURE 3: The specific heat as a function of the ratio of temperature to dipolar coupling parameter for SHA (a), HCPA (b), and FCCA (c). 


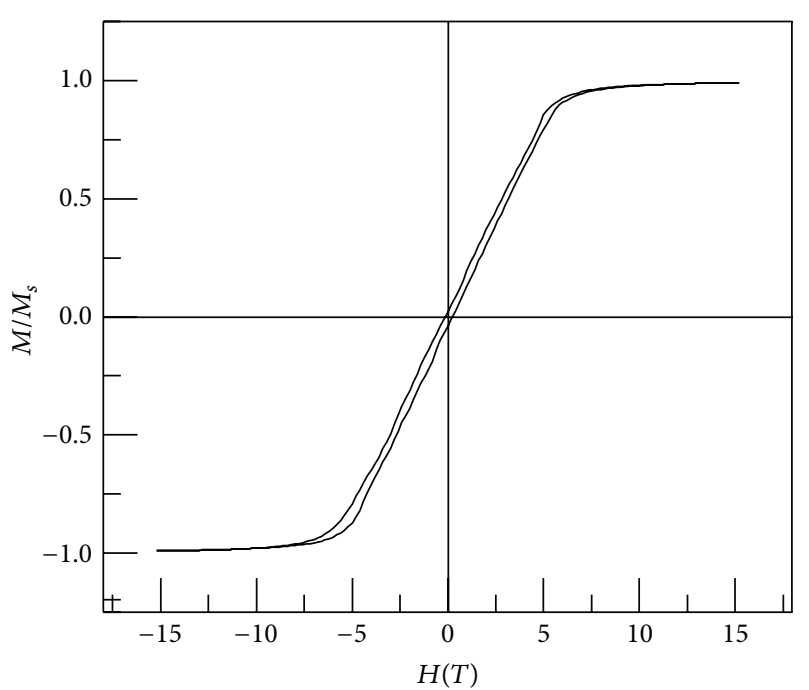

(a)

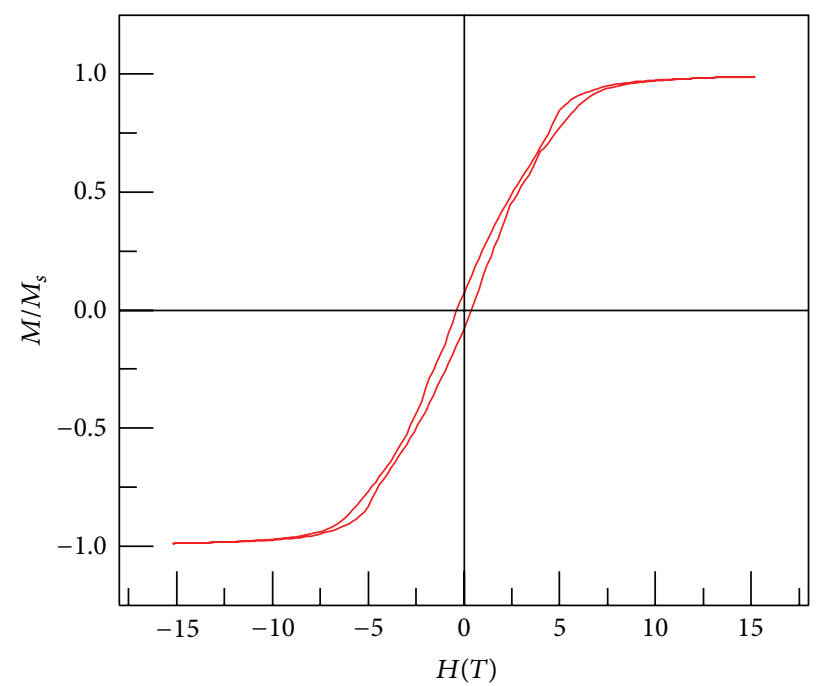

(b)

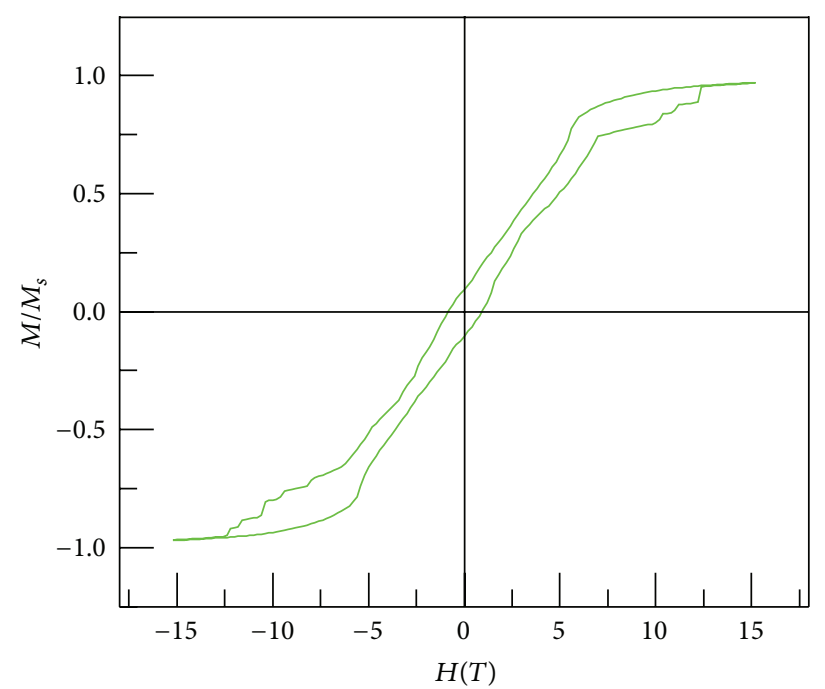

(c)

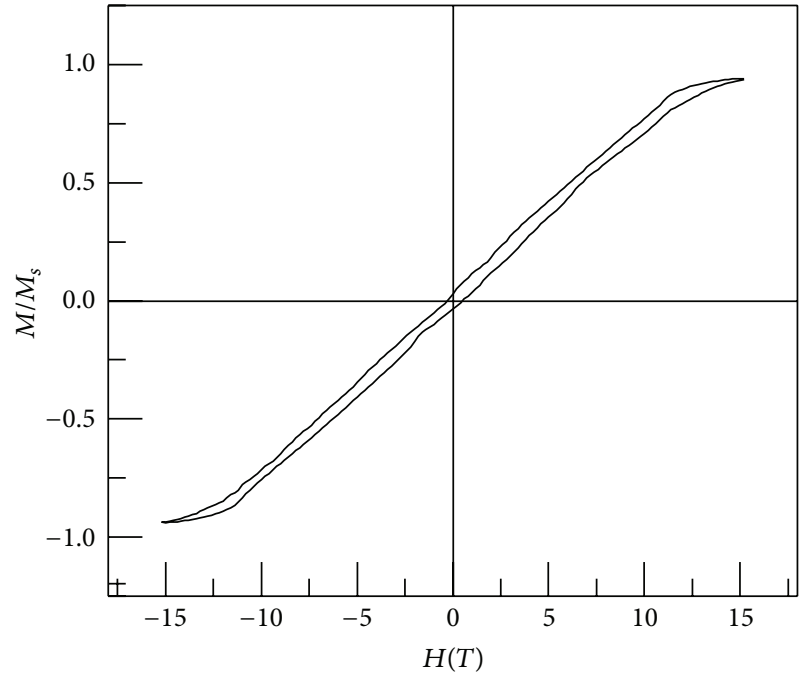

(d)

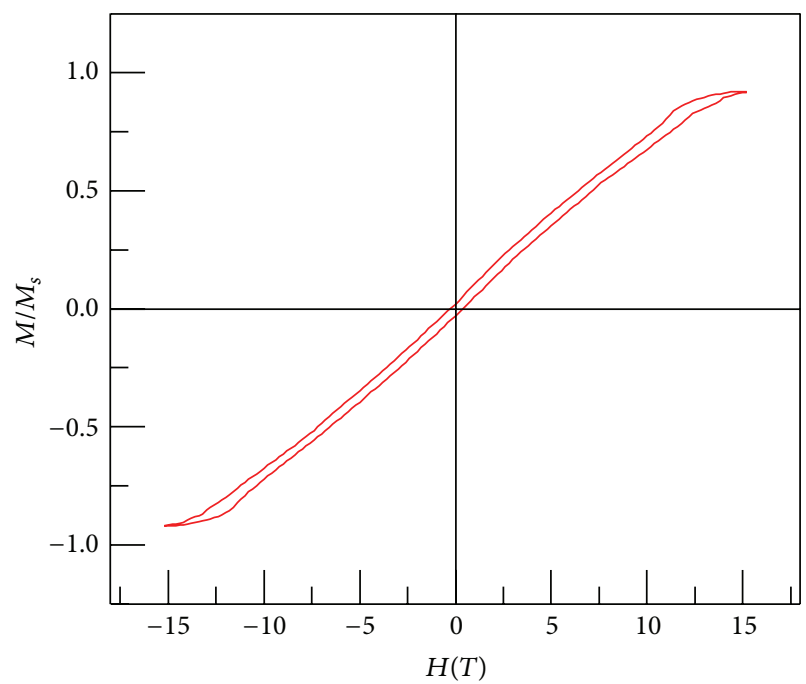

(e)

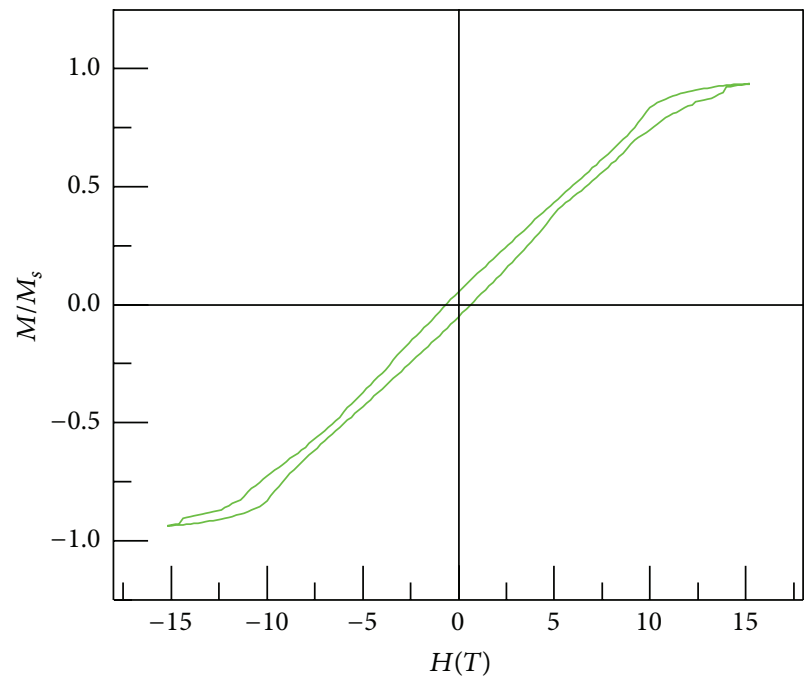

(f)

FIGURE 4: The hysteresis loop for SHA (a), HCPA (b), and FCCA (c) when the dipolar coupling parameter is $1.62 \mathrm{eV}$; the hysteresis loop for SHA (d), HCPA (e), and FCCA (f) with the dipolar coupling parameter of $3.62 \mathrm{eV}$. 
to the positive saturated field $+H_{s}$, so that a full hysteresis loop is completed. The long enough MC steps per spin for each hysteresis loop are used for acquiring the thermal equilibrium. To reduce error, each data point is averaged over 10 independent runs in our calculation.

\section{Results and Discussions}

Figure 2(a) presents the magnetic ground configurations of the simple hexagonal nanomagnet array. Here, we show the magnetic spin configurations of two layers with opposite vortex orders, one clockwise direction and the other counterclockwise direction, which are the repeated unit of the whole magnetic configurations. All spins tend to point in the plane, and the adjacent two layers have antiparallel magnetic ordering. This is due to the fact that the magnetic orientation of each spin is mainly dominated by the dipolar interactions from its six nearest-neighbor spins in the plane, leading to vortex ordering, while the dipolar interactions from two nearest-neighbor spins above and below the plane mainly result in the antiparallel ordering between adjacent two layers.

When the nanomagnets are arranged with the hexagonal close-packed array and face-centered cubic arrays, the magnetic configurations of adjacent three layers are shown in Figures 2(b) and 2(c), respectively. It can be seen from Figure 2(b) that for HCPA three layers exhibit the same vortex orders direction, but the spins at the vortex center are found to tend to orientate out of the plane as a result of strong dipolar interactions derived mainly from six staggered nearest-neighbor spins above and below the plane. Similarly, for FCCA, the same vortex order is also found in each layer (Figure 2(c)), but differently the vortex center exhibits an apparent movement among three layers as well as a slight deviation of the spin orientations from the plane.

In order to understand further the magnetic order transition in these nanomagnet assemblies, we calculate the specific heat for three different nanomagnet arrays. Figure 3 shows the specific heat as a function of the ratio of temperature to dipolar coupling parameter. We find one peak in three types of nanomagnet arrays at $T / D=0.659$, which corresponds to the transition from the vortex order to paramagnetic order. The magnetic transition temperature of three types of nanomagnet assembly is almost the same except that the specific heat curve after transition temperature in SHA decreases more quickly than in HCPA and FCCA.

Figure 4 shows the magnetization reversal of three nanomagnet assemblies at the ground state temperature $(100 \mathrm{~K}$ here) in the case of two different dipolar coupling parameters, that is, two difference space distances between nanomagnets. Figures 4(a)-4(c) are the magnetic hysteresis loop of three nanomagnet arrays when the dipolar coupling parameter is $1.62 \mathrm{eV}$ for SHA, HCPA, and FCCA, respectively, while Figures $4(\mathrm{~d})-4(\mathrm{f})$ are results from $3.62 \mathrm{eV}$. It can be seen that the magnetization saturates at high magnetic field, and the saturated magnetic field is smaller for the loose nanomagnet array than the close nanomagnet array as a consequence of competing interactions between dipolar interactions and field interactions. The shape of hysteresis loop for SHA and HCPA is very similar for two dipolar parameters. For FCCA, some slight jumps occur in the hysteresis loop due to the strong dipolar interactions between each spin and its 12 nearestneighbor spins, and in this case the saturated magnetic field is larger than that of SHA and HCPA.

\section{Conclusions}

In summary, we have studied the magnetic ground state and magnetization reversal properties of three types of dipolecoupled nanomagnet arrays, that is, simple hexagonal array, hexagonal close-packed array, and face-centered array, in three-dimensional space by the Monte Carlo method. The magnetic ground state is found to exhibit the vortex order for three different nanomagnet arrays, which is attributed to the long-range dipolar interactions. The magnetic transition from the vortex order to paramagnetic order occurs when the ratio of temperature to dipolar coupling parameter is 0.659 , and the magnetic transition temperature is almost the same for three types of nanomagnet arrays. By comparing with the simple hexagonal nanomagnet array and close-packed hexagonal nanomagnet array, we find that the saturated field and remanence are larger for the face-centered cubic nanomagnet array.

\section{Conflict of Interests}

The authors declare that there is no conflict of interests regarding the publication of this paper.

\section{Acknowledgment}

This work is supported by the National Nature Science Foundation of China (Grant no. 11204064 and U1304518).

\section{References}

[1] S. Bedanta, A. Barman, W. Kleemann, O. Petracic, and T. Seki, "Magnetic nanoparticles: a subject for both fundamental research and applications," Journal of Nanomaterials, vol. 2013, Article ID 952540, 22 pages, 2013.

[2] D. A. Allwood, G. Xiong, C. C. Faulkner, D. Atkinson, D. Petit, and R. P. Cowburn, "Magnetic domain-wall logic," Science, vol. 309, no. 5741, pp. 1688-1692, 2005.

[3] A. Imre, G. Csaba, L. Ji, A. Orlov, G. H. Bernstein, and W. Porod, "Majority logic gate for magnetic quantum-dot cellular automata," Science, vol. 311, no. 5758, pp. 205-208, 2006.

[4] B. Lenk, H. Ulrichs, F. Garbs, and M. Münzenberg, "The building blocks of magnonics," Physics Reports, vol. 507, no. 4-5, pp. 107-136, 2011.

[5] M. Vacca, M. Graziano, J. C. Wang et al., "Nanomagnet logic: an architectural level overview," in Field-Coupled Nanocomputing: Paradigms, Progress, and Perspectives, vol. 8280 of Lecture Notes in Computer Science, pp. 223-256, Springer, Berlin, Germany, 2014.

[6] R. P. Cowburn, A. O. Adeyeye, and M. E. Welland, "Controlling magnetic ordering in coupled nanomagnet arrays," New Journal of Physics, vol. 1, no. 1, article 16, 1999. 
[7] R. F. Wang, C. Nisoli, R. S. Freitas et al., "Artificial spin ice in a geometrically frustrated lattice of nanoscale ferromagnetic islands," Nature, vol. 439, pp. 303-306, 2006.

[8] M. Ewerlin, D. Demirbas, F. Brüssing et al., "Magnetic dipole and higher pole interaction on a square lattice," Physical Review Letters, vol. 110, no. 17, Article ID 177209, 2013.

[9] A. Kumari, S. Sarkar, J. F. Pulecio, D. K. Karunaratne, and S. Bhanja, "Study of magnetization state transition in closely spaced nanomagnet two-dimensional array for computation," Journal of Applied Physics, vol. 109, no. 7, Article ID 07E513, 2011.

[10] S. Rajaram, D. K. Karunaratne, S. Sarkar, and S. Bhanja, "Study of dipolar neighbor interaction on magnetization states of nano-magnetic disks," IEEE Transactions on Magnetics, vol. 49, no. 7, pp. 3129-3132, 2013.

[11] A. J. Bennett and J. M. Xu, "Simulating collective magnetic dynamics in nanodisk arrays," Applied Physics Letters, vol. 82, no. 15, pp. 2503-2505, 2003.

[12] Y. Li and B.-G. Liu, "Long-range ferromagnetism in onedimensional monatomic spin chains," Physical Review B, vol. 73, no. 17, Article ID 174418, 2006.

[13] Y. Li, Y. F. Li, and T. X. Wang, "The size and shape dependence of ferromagnetism in nanomagnets," Journal of Nanomaterials, vol. 2012, Article ID 897203, 6 pages, 2012.

[14] Y. Li, T. X. Wang, and G. D. Liu, "Thermodynamic and magnetic properties in two artificial frustrated lattices," Physics Letters A, vol. 377, no. 25-27, pp. 1655-1660, 2013.

[15] G.-W. Chern, C. Reichhardt, and C. Nisoli, "Realizing threedimensional artificial spin ice by stacking planar nano-arrays," Applied Physics Letters, vol. 104, no. 1, Article ID 013101, 2014.

[16] D. Givord, Q. Lu, and M. F. Rossignol, Science and Technology of Nanostructured Materials, edited by G. C. Hadjipanayis and G. A. Prinz, PlenumPress, New York, NY, USA, 1991.

[17] N. Q. Metropolis, A. W. Rosenbluth, M. N. Rosenbluth, A. H. Teller, and E. Teller, "Equation of state calculations by fast computing machines," The Journal of Chemical Physics, vol. 21, no. 6, pp. 1087-1092, 1953.

[18] J. E. Gubernatis, "Marshall rosenbluth and the metropolis algorithm," Physics of Plasmas, vol. 12, Article ID 057303, 2005.

[19] M. E. J. Newman and G. T. Barkema, Monte Carlo Methods in Statistical Physics, Clarendon Press, Oxford, UK, 1999. 

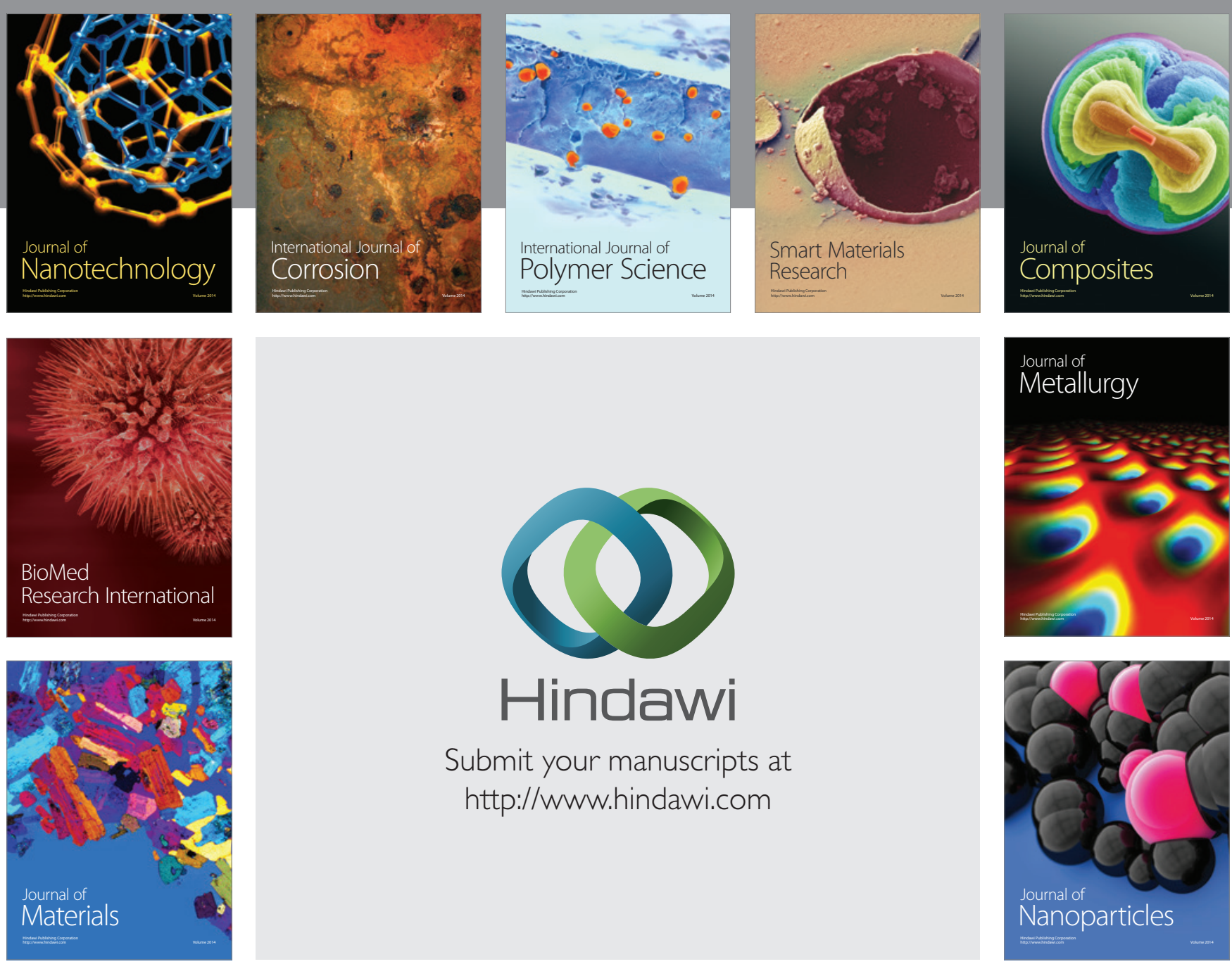

Submit your manuscripts at http://www.hindawi.com
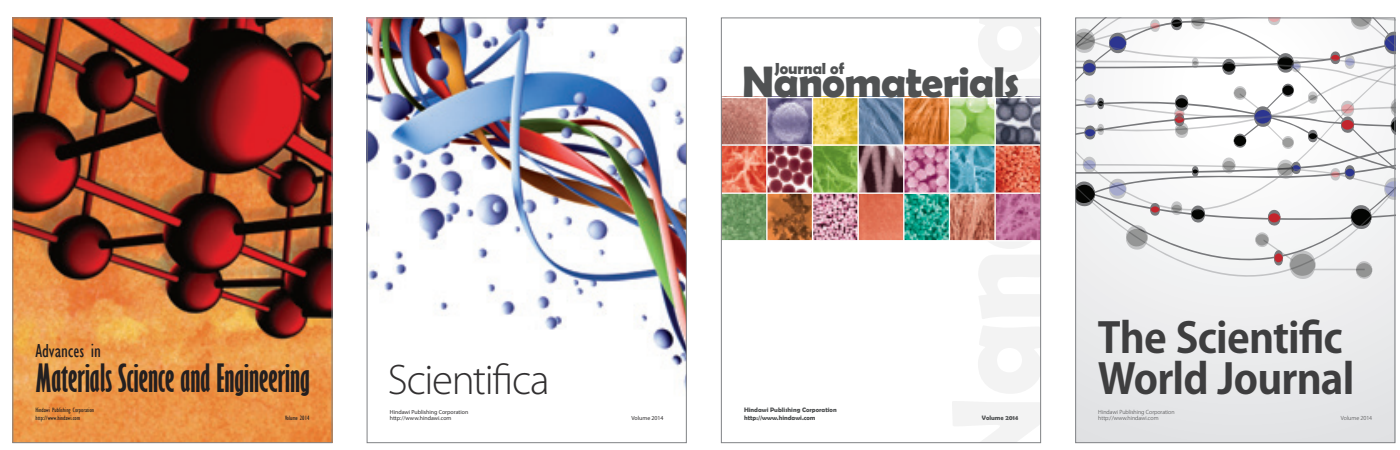

\section{The Scientific World Journal}
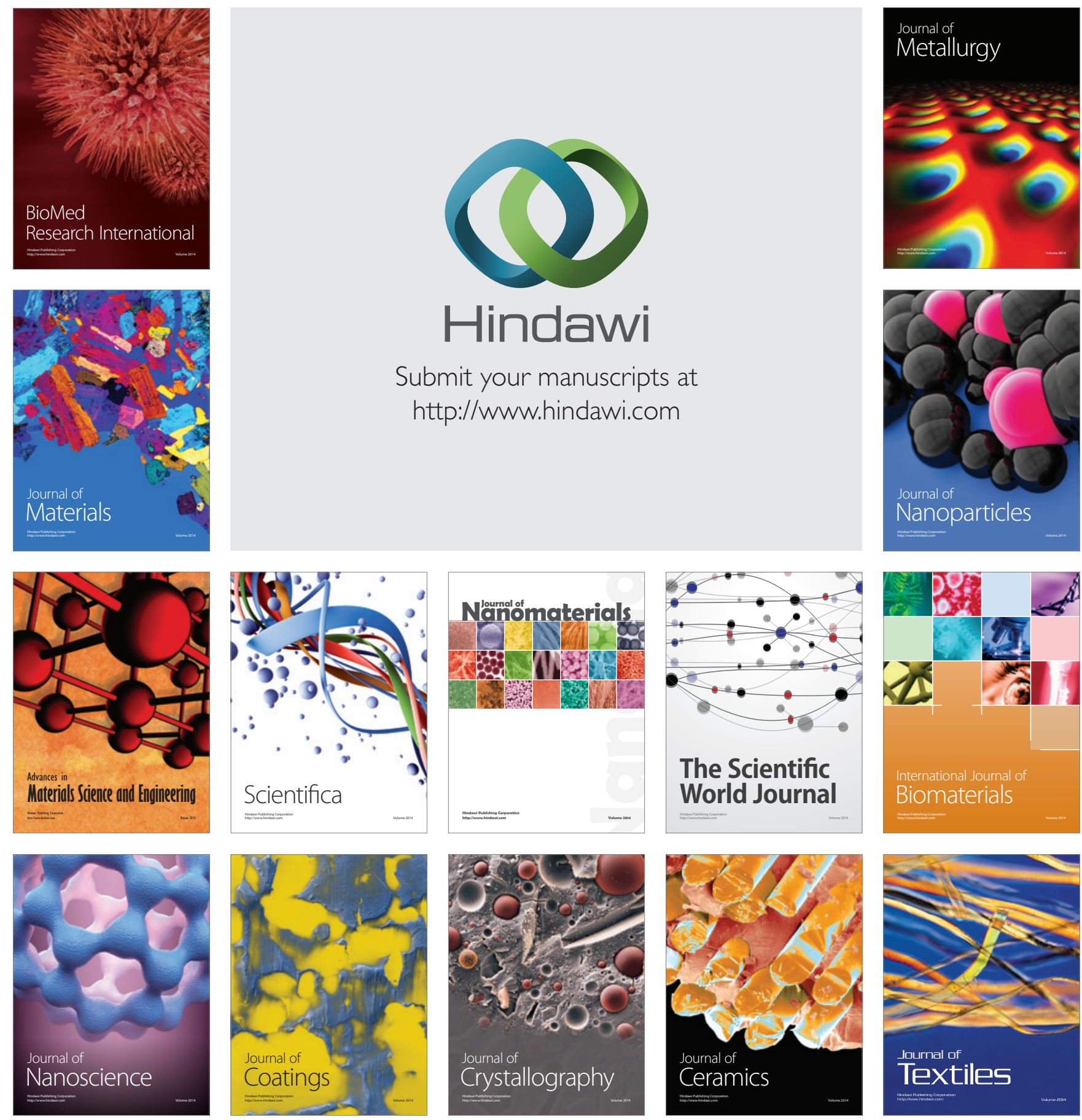